\title{
Enhanced Rashba effect for hole states in a quantum dot
}

\author{
Aram Manaselyan and Tapash Chakraborty \\ Department of Physics and Astronomy, University of Manitoba, Winnipeg, Canada R3T 2N2 \\ PACS 73.21.La - Quantum dots \\ PACS 71.70.Ej - Spin-orbit coupling \\ PACS 75.75. $+\mathrm{a}-$ Magnetic properties of nanostructures
}

\begin{abstract}
The effect of Rashba spin-orbit (SO) interaction on the hole states in a quantum dot is studied in the presence of an external magnetic field. We demonstrate here that the Rashba SO coupling has a profound effect on the energy spectrum of the holes revealing level repulsions between the states with the same total momentum. We also show that the resulting spin-orbit gap is much larger than the corresponding one for the electron energy levels in a quantum dot. Inter-hole interactions only marginally reduce the spin-orbit gap. This enhanced Rashba effect would manifest itself in the tuneling current which depends on the spin-orbit coupling strength.
\end{abstract}

Introduction. - Semiconductor quantum dots are the nanoscale zero-dimensional systems with discrete energy levels, much like in atoms (and hence the popular name, artificial atoms [1]). They have one great advantage that their shape and the number of electrons in those ' systems can be controlled externally and as a result, they have been the subject of intense research in recent years. ' They are particularly promising as components of futuristic devices for quantum information processing [2] and , for coherent spin transport [3]. The spin states of these systems are ideal for applications because of their relative insensitivity to electrical noise in a device environment [4]. One proposed mechanism for coherent spin manipulation ' in quantum nanostructures is via the Rashba spin-orbit ( $\mathrm{SO}$ ) coupling $[5,6]$. The $\mathrm{SO}$ interaction can arise in a quantum dot due to confinement and lack of inversion symmetry of the nanostructure which creates a local electric field perpendicular to the electron plane $[7,8]$. The SO coupling strength can be varied by changing the asymmetry of the quantum structure with an external electric field. The magnetic field effects on the properties of lowdimensional systems, such as quantum wells and quantum dots with the Rashba interaction has been reported in experiments [9] and theory [10]. In our work on Rashba effects in electron dots [11], we found multiple level crossings and level repulsions that resulted from the interplay between the Zeeman and the SO couplings. Level anticrossings observed in quantum nanostructures have been attributed to the presence of SO coupling in those systems [12]. However, studies of the Rashba effect on quan- tum dots as yet, are limited only to the case of electrons as charge carriers.

The importance of holes in semiconductor spintronics is well documented [13] in the literature. Some theoretical results about hole states in quantum dots have been reported earlier [14-16]. Experiments on hole levels in quantum dots have also been reported recently [17-19]. Rashba effect is expected to be stronger in $p$-type quantum wells [20]. Interestingly, for holes the Dresselhaus effect (due to bulk inversion asymmetry) is small compared to the Rashba effect [21]. In this Letter, we report on our studies involving the hole levels in planar quantum dots with Rashba SO interactions. We find that the SO gap at the anticrossings of the energy levels is much larger than those for the case of electrons. Inter-particle interactions (Coulomb type) reduce the gap somewhat, but it is still orders of magnitude larger than the corresponding ones for electrons.

The Rashba effect for holes is different from that for the electrons. It is well known that the electron Rashba coefficient increases nearly linearly with an increase of the electric field. But the two-dimensional heavy hole systems in single heterostructures exhibit a decrease of Rashba SO splitting with an increase of the electric field [22]. The effect for a two-dimensional light hole system is however the same as that for the electrons. Determination of the Rashba coefficient for holes was reported for the InP quantum wires [23] where the band mixing was taken into account. The result was that as the electric field increases, the hole Rashba coefficient increases at first, then 
decreases. There is also a critical electric field for which the hole Rashba coefficient vanishes. No such calculations have been reported as yet, for the hole Rashba coefficients in quantum dots. However electric field still remains an useful tool to control the Rashba coefficient.

Theory. - Let us consider the hole states in a InAs/GaAs cylindrical quantum dot in the presence of an external magnetic field directed along the $z$ axis. Taking into account only the $\Gamma_{8}$ states which correspond to the states with hole spin $J=3 / 2$, we can construct the single-hole Hamiltonian of the system as

$$
\mathcal{H}=\mathcal{H}_{L}+\mathcal{H}_{Z}+V_{\text {conf }}(\rho, z)+\mathcal{H}_{\mathrm{SO}} .
$$

Here $\mathcal{H}_{L}$ is the Luttinger hamiltonian in axial representation obtained with the four-band $\mathbf{k} \cdot \mathbf{p}$ theory $[16,24]$

$$
\mathcal{H}_{L}=\frac{1}{2 m_{0}}\left(\begin{array}{cccc}
\mathcal{H}_{h} & R & S & 0 \\
R^{*} & \mathcal{H}_{l} & 0 & S \\
S^{*} & 0 & \mathcal{H}_{l} & -R \\
0 & S^{*} & -R^{*} & \mathcal{H}_{h}
\end{array}\right)
$$

where

$$
\begin{aligned}
\mathcal{H}_{h} & =\left(\gamma_{1}+\gamma_{2}\right)\left(\Pi_{x}^{2}+\Pi_{y}^{2}\right)+\left(\gamma_{1}-2 \gamma_{2}\right) \Pi_{z}^{2}, \\
\mathcal{H}_{l} & =\left(\gamma_{1}-\gamma_{2}\right)\left(\Pi_{x}^{2}+\Pi_{y}^{2}\right)+\left(\gamma_{1}+2 \gamma_{2}\right) \Pi_{z}^{2},
\end{aligned}
$$

$R=2 \sqrt{3} \gamma_{3}$ i $\Pi_{-} \Pi_{z}, \quad S=\sqrt{3} \gamma \Pi_{-}^{2}, \quad \gamma=\frac{1}{2}\left(\gamma_{2}+\gamma_{3}\right)$, and $\boldsymbol{\Pi}=\mathbf{p}-\frac{e}{c} \mathbf{A}, \quad \Pi_{ \pm}=\Pi_{x} \pm i \Pi_{y} \cdot \gamma_{1}, \gamma_{2}$ and $\gamma_{3}$ are the Luttinger parameters and $m_{0}$ is the free electron mass. The Hamiltonian is presented in the hole picture, where the energies are positive, $\mathbf{A}$ is the usual symmetric gauge vector potential. The Zeeman Hamiltonian $\mathcal{H}_{Z}$ is a $4 \times 4$ diagonal matrix with diagonal elements $-\kappa \mu_{B} B j_{z}$, where $\kappa$ is the fourth Luttinger parameter, $\mu_{B}$ is the Bohr magneton and $j_{z}$ is the projection of the hole spin on the $z$ axis $\left(j_{z}=3 / 2,1 / 2,-1 / 2,-3 / 2\right)$.

We choose the lateral confinement potential of the dot as parabolic with oscillator energy $\hbar \omega_{0}$. This can be justified from the energies of far-infrared absorption on such dots which show only a weak dependence on the electron occupation [25]. We also take into account the confinement potential in the $z$ direction as a rectangular well of width $L$. Just as for electron states in parabolic quantum dots [11], the term $\mathcal{H}_{\mathrm{SO}}$ describes the Rashba spin-orbit coupling [5] due to the inhomogeneous potential that confines the holes in a plane

$$
\mathcal{H}_{\mathrm{SO}}=\frac{\alpha}{\hbar}[\mathbf{J} \cdot \boldsymbol{\Pi}]_{z}=\frac{\alpha}{\hbar}\left(J_{x} \Pi_{y}-J_{y} \Pi_{x}\right),
$$

where $\alpha$ is the Rashba coefficient for the holes and $J_{x}, J_{y}$ are the hole spin matrices

$$
J_{x}=\left(\begin{array}{cccc}
0 & \mathrm{i} \sqrt{3} & 0 & 0 \\
-\mathrm{i} \sqrt{3} & 0 & 2 i & 0 \\
0 & -2 i & 0 & \mathrm{i} \sqrt{3} \\
0 & 0 & -\mathrm{i} \sqrt{3} & 0
\end{array}\right),
$$

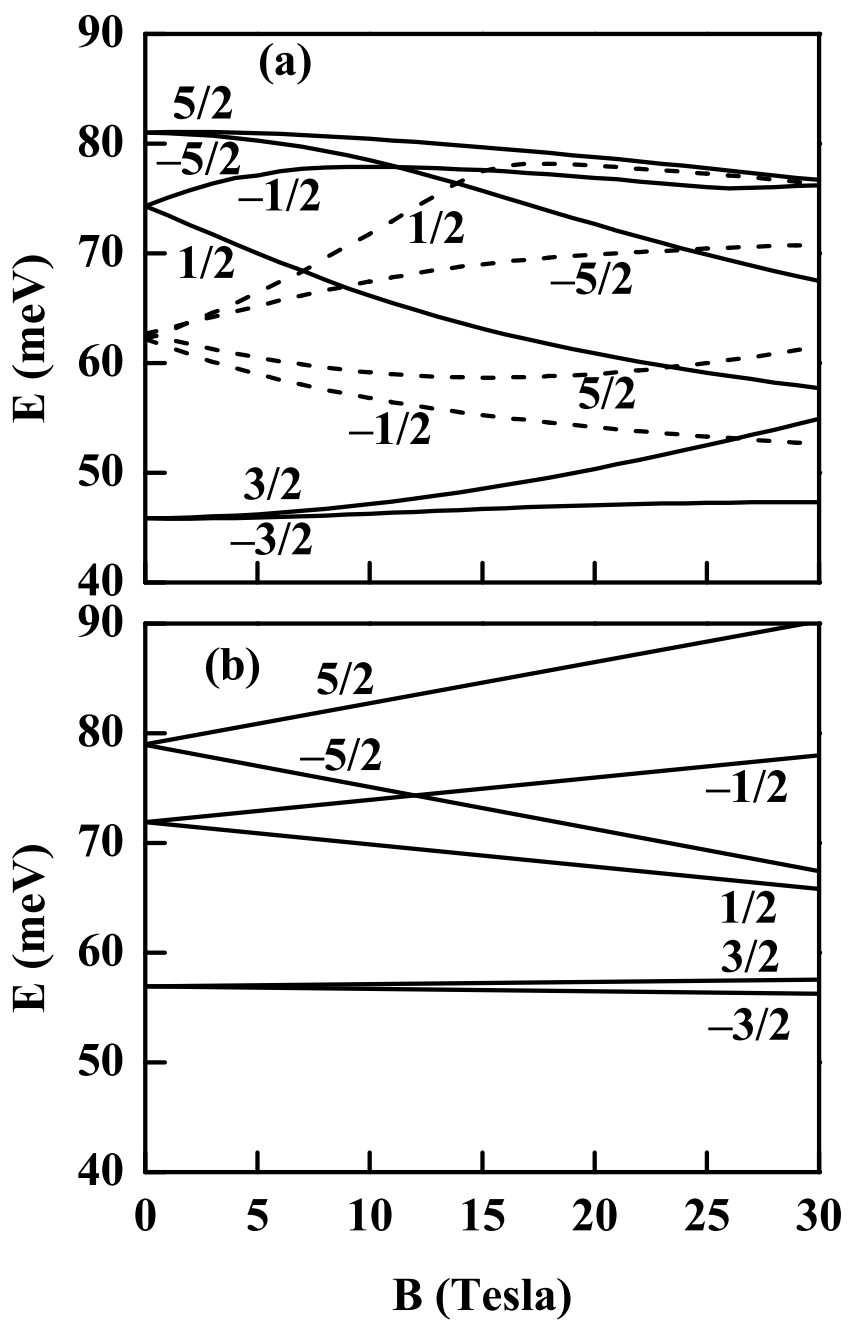

Fig. 1: Magnetic field dependence of single hole energy levels in the absence of spin-orbit coupling. (a) Our theoretical results: Solid lines have even parity and dashed lines have odd parity. (b) Results derived from the experimental data [18]. The curves are labelled by their corresponding total momentum $F_{z}$.

$$
J_{y}=\left(\begin{array}{cccc}
0 & \sqrt{3} & 0 & 0 \\
\sqrt{3} & 0 & 2 & 0 \\
0 & 2 & 0 & \sqrt{3} \\
0 & 0 & \sqrt{3} & 0
\end{array}\right)
$$

It is well known [26] that in two-dimensional systems for heavy holes the Rashba interaction is cubic in momentum because the linear term is zero and we have to keep the higher order terms. In the case of quantum wires and quantum dots it is not possible to separate the heavy and light hole states any more due to strong band mixing effects. Then the linear term in Rashba interaction will not be zero and we can neglect the cubic terms which are much smaller. The final form of the spin-orbit coupling 
hamiltonian is then

$$
\mathcal{H}_{\mathrm{SO}}=-\frac{\alpha}{\hbar}\left(\begin{array}{cccc}
0 & \sqrt{3} \Pi_{-} & 0 & 0 \\
\sqrt{3} \Pi_{+} & 0 & 2 \Pi_{-} & 0 \\
0 & 2 \Pi_{+} & 0 & \sqrt{3} \Pi_{-} \\
0 & 0 & \sqrt{3} \Pi_{+} & 0
\end{array}\right) .
$$

The Hamiltonian (1) is rotationally invariant. Therefore it will be useful to introduce the total momentum $\mathbf{F}=\mathbf{J}+\mathbf{L}$, where $\mathbf{J}$ is the angular momentum of the band edge Bloch function, and $\mathbf{L}$ is the envelop angular momentum. Since the projection of the total momentum $F_{z}$ is a constant of motion, we can find simultaneous eigenstates for (11) and $F_{z}$ [27]. Therefore for a given value of $F_{z}$ a general hole state can be written as [16]

$$
\Psi_{F_{z}}(\rho, \theta, z)=\sum_{j_{z}} \psi_{j_{z}}(\rho, z) \mathrm{e}^{\mathrm{i}\left(F_{z}-j_{z}\right) \theta}\left|3 / 2, j_{z}\right\rangle,
$$

where $\left|3 / 2, j_{z}\right\rangle$ is the Bloch function, $\psi_{j_{z}}(\rho, z)$ is the envelop function and instead of the angular momentum quantum number $l=0, \pm 1, \ldots$, we write its allowed values $l=F_{z}-j_{z}$.

First, we solve the problem only for $\mathcal{H}_{h}$ which corresponds to the state $|3 / 2,3 / 2\rangle$. For the in-plane problem we get the equation of a two-dimensional harmonic oscillator in a magnetic field, the solution of which is

$$
f_{n l}(\rho, \theta)=C_{n l}\left(\frac{\mathrm{i} \rho}{a}\right)^{|l|} \mathrm{e}^{-\rho^{2} / 2 a^{2}} L_{n}^{|l|}\left(\frac{\rho^{2}}{a^{2}}\right) \mathrm{e}^{\mathrm{i} l \theta} .
$$

In Eq. (41) $L_{n}^{l}(x)$ is the generalized Laguerre polynomial, $\quad C_{n l}=\frac{1}{a}\left[\frac{n !}{\pi(n+|l|) !}\right]^{\frac{1}{2}}, \quad a=\left[\frac{\hbar\left(\gamma_{1}+\gamma_{2}\right)}{m_{0} \omega}\right]^{\frac{1}{2}}$, $\omega=\sqrt{\omega_{0}^{2}+\frac{1}{4} \omega_{c}^{2}}, \quad \omega_{c}=e B\left(\gamma_{1}+\gamma_{2}\right) / m_{0} c$ is the cyclotron frequency, and $n$ is the radial quantum number $(n=0,1, \ldots)$. The energy levels are given by $E_{n l}=2 \hbar \omega\left(n+1 / 2(|l|+1)-l \omega_{c} / 4 \omega\right)$. For the $z$ part of the problem we can use the results for a one-dimensional rectangular quantum well

$$
g_{s}(z)=\sqrt{\frac{2}{L}} \sin \left[\frac{s \pi}{L}\left(z+\frac{L}{2}\right)\right],
$$

with energies $E_{s}=\pi^{2} \hbar^{2} s^{2}\left(\gamma_{1}-2 \gamma_{2}\right) / 2 m_{0} L^{2}, \quad s=$ $1,2,3, \ldots$

It is logical to seek the eigenfunctions of Hamiltonian (11) as an expansion with the basis functions (4) and (5).

$$
\Psi_{F_{z}}(\rho, \theta, z)=\sum_{n, s, j_{z}} C\left(n, s, j_{z}\right) f_{n, F_{z}-j_{z}}(\rho, \theta) g_{s}(z)
$$

or in the corresponding spinor representation.

The matrix elements of the Hamiltonian (1) can then be evaluated analytically. All energies and wave functions for the single hole system are evaluated numerically using the exact diagonalization scheme [11]. If we choose $n_{\max }$ number of in-plane basis states and $s_{\max }$ number of basis
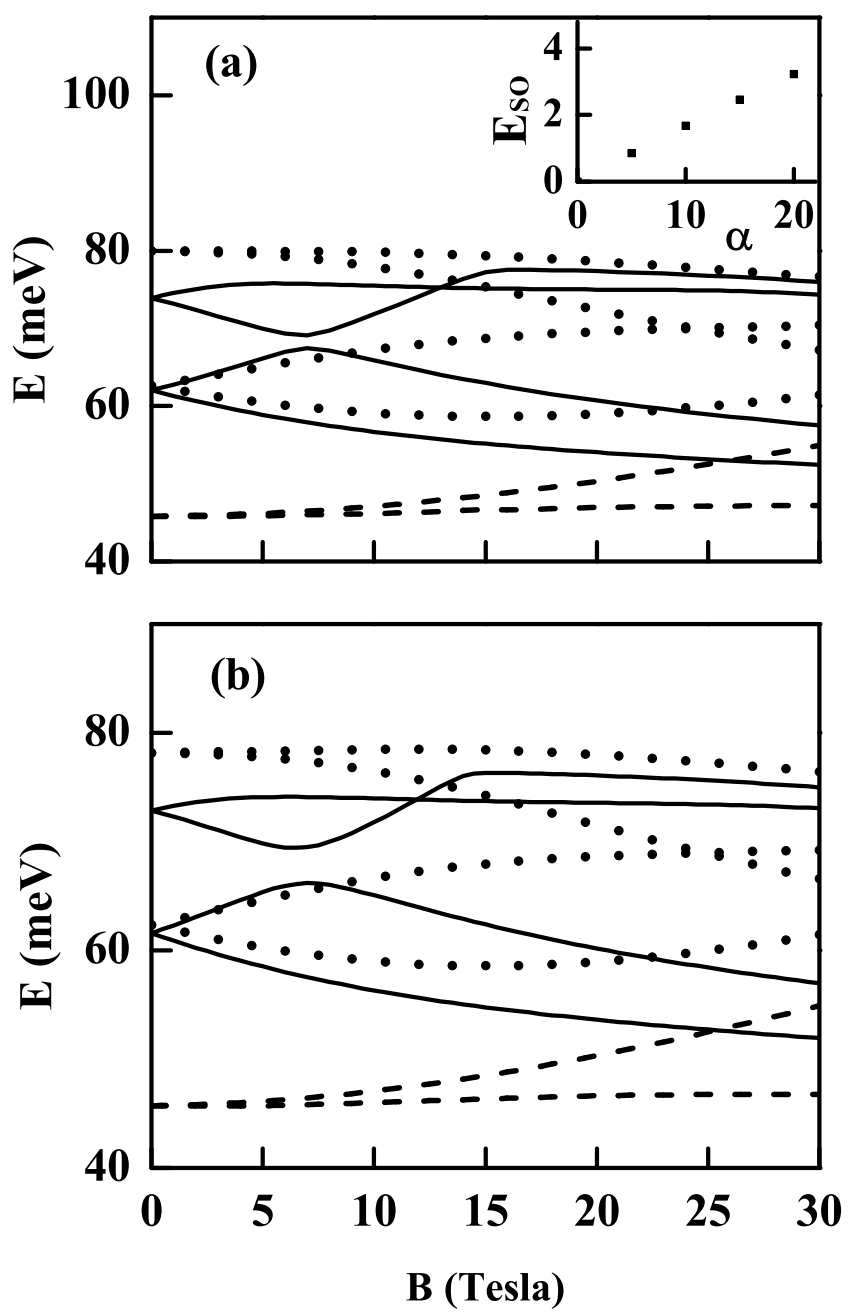

Fig. 2: Dependence of the single hole energy levels on the magnetic field taking into account the spin-orbit coupling. (a) $\alpha=10 \mathrm{meV} \mathrm{nm}$. (b) $\alpha=20 \mathrm{meV} \mathrm{nm}$. Solid lines: $F_{z}= \pm 1 / 2$, dashed lines: $F_{z}= \pm 3 / 2$, dotted lines: $F_{z}= \pm 5 / 2$. The spinorbit gap $E_{\mathrm{SO}}$ vs $\alpha$ is shown as inset.

states for the $z$ direction, we get a matrix of the order $N=$ $4 n_{\max } s_{\max }$. In that matrix each element of the Luttinger Hamiltonian has its submatrix of order $n_{\max } s_{\max }$. The submatrix of $\mathcal{H}_{h}$ will be diagonal, but the submatrix of $\mathcal{H}_{l}$ will not be so because we are using the eigenfunctions of $\mathcal{H}_{h}$ as the basis functions. We have also considered an interacting two-hole system, where the interaction matrix elements are formally same as those for electrons $[1,11]$.

Discussion of results. - All computations were carried out for the InAs/GaAs quantum dot with parameters $\gamma_{1}=11.01, \gamma_{2}=4.18, \gamma_{3}=4.84, \kappa=1.2$ and the dielectric constant, $\varepsilon=12.4$ [28]. The height of the dot is taken as $L=4.5 \mathrm{~nm}$ and the in-plane confinement energy $\hbar \omega_{0}=20 \mathrm{meV}$ which seems to be very reasonable $[18,29]$.

First we compare our results for $\alpha=0$ with the results of the experimental work [18] where the dependence of the single hole energy levels on the magnetic field was evalu- 
ated using the capacitance-voltage and polarized photoluminescence spectroscopy measurements in high magnetic fields. In Fig. 1 the magnetic field dependence of the single hole energy levels are presented for different values of the total momentum $F_{z}$. Our results are shown in Fig. 1(a), and the ones derived by the experimental group [18] are shown in Fig. 1(b). In both figures the ground state corresponds to $F_{z}=-3 / 2$, which lies below the state with $F_{z}=3 / 2$ for all values of the magnetic field. Clearly, in Fig. 1(a) there are some levels with $F_{z}= \pm 1 / 2$ and $F_{z}= \pm 5 / 2$ (dashed lines) that are absent in Fig. 1 (b). The solid lines in Fig. 1(a) have even parity while the dashed lines have odd parity [16]. We believe that the absence of the lines in Fig. 1(b), but presence in our results is due to the parity selection rule which in the photoluminescence spectroscopy experiment does not allow the states presented as dashed lines in Fig. 1(a). In Fig. 1(a) the energies of the states $F_{z}= \pm 5 / 2$ with even parity are found to decrease with increasing magnetic field. This is in contrast to the experimental data [Fig. 1(b)], where the energy of the state $F_{z}=5 / 2$ increases with increasing magnetic field. This difference in the magnetic behavior is due to particular assumptions in [18] that are employed to derive the hole energy from the experimental data. However, what is crucial here is the separation between the two curves that is very similar in both cases. One of the most interesting experimental results of [18] is the level crossing point around 12 Tesla between the states $F_{z}=-1 / 2$ and $F_{z}=-5 / 2$, which is also present in our results in Fig.1(a). Overall, our results for the hole energy levels (in the absence of Rashba effect) are in quite good agreement with the results of [18].

In Fig. 2, the magnetic field dependence of the singlehole energy levels is depicted for different values of the total momentum $F_{z}$, where the Rashba spin-orbit coupling is taken into account, with $\alpha=10 \mathrm{meV} \mathrm{nm} \mathrm{[Fig.} \mathrm{22(a)]} \mathrm{and}$ $\alpha=20 \mathrm{meV} \mathrm{nm} \mathrm{[Fig.} \mathrm{2(b)].} \mathrm{In} \mathrm{the} \mathrm{absence} \mathrm{of} \mathrm{the} \mathrm{spin} \mathrm{or-}$ bit coupling (Fig. 1(a)) the two lowest energy states with total momentum $F_{z}=1 / 2$ cross at a finite magnetic field near 7 Tesla. Spin orbit interaction mixes the states with same total momentum $F_{z}$ and different parities. Hence in Fig. 2(a), instead of a level crossing we actually have an 'anticrossing' with an energy gap of $E_{\mathrm{SO}} \approx 2 \mathrm{meV}$; we can no longer separate the states by parity. With an increase of the spin-orbit coupling the size of the gap increases and for $\alpha=20 \mathrm{meV} \mathrm{nm} \mathrm{(Fig.} \mathrm{2(b))} \mathrm{the} \mathrm{gap} \mathrm{is} E_{\mathrm{SO}} \approx 4 \mathrm{meV}$ [see inset in Fig. 2(a)]. A spin-orbit gap was also found earlier [30] for the electron states in quantum dots and quantum rings, but the gap was much smaller in comparison to the present case. A comparison of Fig. 2(a) and Fig. 2(b) clearly reveals that the effect of the spin-orbit coupling is very small for the ground states with $F_{z}= \pm 3 / 2$. The reason of this behavior is that the dominant components of the states $F_{z}=3 / 2$ and $F_{z}=-3 / 2$ are those corresponding to $j_{z}=3 / 2$ and $j_{z}=-3 / 2$ respectively. But the spin-orbit Hamiltonian (3) does not mix those states. Therefore we conclude that the effect of Rashba spin-orbit
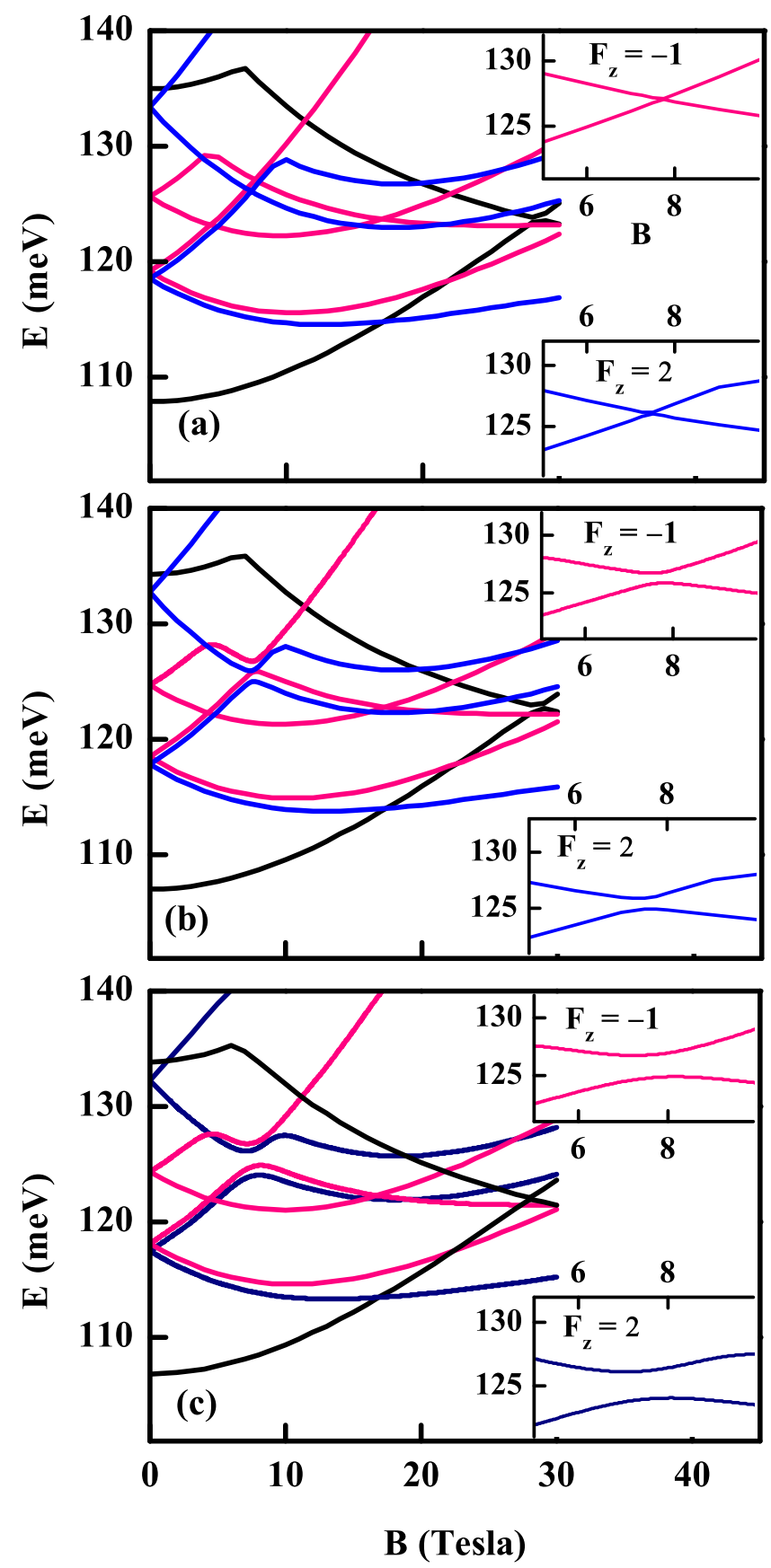

Fig. 3: Dependence of the two holes (interacting) energy levels on the magnetic field for total momentum $F_{z}=0$ (black), $F_{z}= \pm 1$ (pink) and $F_{z}= \pm 2$ (blue). (a) $\alpha=0$. (b) $\alpha=10$ $\mathrm{meV} \mathrm{nm}$. (c) $\alpha=20 \mathrm{meV} \mathrm{nm}$. Insets show the level crossing (a) and level repulsion (b,c) gaps for $F_{z}=-1$ and $F_{z}=2$.

coupling is much stronger and more important for the hole states in the valence band. It mixes states with even and odd parity, removes the crossing points, and introduces level repulsion between the states with the same total momemntum $F_{z}$.

In Fig. 3 the magnetic field dependence of the two-hole (interacting) energy levels is depicted for different values 
of the total momentum $F_{z}=0, \pm 1, \pm 2$. For small values of the magnetic field, the ground state has total momentum $F_{z}=0$. But starting from a field of 17 Tesla it changes to $F_{z}=-2$. In the absence of spin-orbit coupling [Fig. [3(a)] we still have several crossing points between the levels with same total momentum. Spin-orbit interaction [Fig. 3(b) and (c)] again mixes those states and instead of level crossings we now have 'anticrossings'. Comparing with the single-hole states we notice that the Coulomb interaction reduces the values of the level repulsion gaps somewhat, but they are still orders of magnitude larger than those for interacting electrons in a quantum dot [11]. Clearly, the Rashba effect has a much more profound influence on hole quantum dots than that for electrons which would manifest itself in a measurably quantity, such as tunneling current in the dot [30].

$$
* * *
$$

The work was supported by the Canada Research Chairs Program and the NSERC Discovery Grant.

\section{REFERENCES}

[1] T. Chakraborty, Quantum Dots (North-Holland, Amsterdam, 1999); C. Schüller, Inelastic Light Scattering of Semiconductor Nanostructures (Springer, Berlin, 2006), Ch. 5.

[2] A. Imamoglu et al., Phys. Rev. Lett. 83 (1999) 4204.

[3] D.D. Awschalom, D. Loss, and N. Samarth (Eds.), Semiconductor Spintronics and Quantum Computation (Springer, 2002); D. Grundler, Phys. World 15 (2002) 39; T. Chakraborty and K. Hall (Eds.), Physics in Canada 63 (2007) 57.

[4] S. Bandyopadhyay, Phys. Rev. B 61 (2000) 13813; J.P. Leburton, et al., Microelectron. J. 34 (2003) 485.

[5] Y.A. Bychkov, E.I. Rashba, J. Phys. C 17 (1984) 6039.

[6] T. Dietl, D.D. Awschalom, M. Kaminska, and H. Ohno, (Eds.) Spintronics (Elsevier, Amsterdam 2008).

[7] I.L. Aleiner, and V.I. Falko, Phys. Rev. Lett. 87 (2001) 256801.

[8] M. Governale, Phys. Rev. Lett. 89 (2002) 206802.

[9] J. Nitta, et al., Phys. Rev. Lett. 78 (1997) 1335; T. Koga, et al., Physica E 13 (2002) 542; D. Grundler, Phys. Rev. Lett. 84 (2000) 6074.

[10] C.F. Destefani, S.E. Ulloa, Phys. Rev. B 71 (2005) 161303.

[11] T. Chakraborty, and P. Pietiläinen, Phys. Rev. Lett. 95 (2005) 136603; P. Pietiläinen, and T. Chakraborty, Phys. Rev. B 73 (2006) 155315.

[12] W. Desrat, et al., Phys. Rev. B 71 (2005) 153314.

[13] A.M. Nazmul, et al., Phys. Rev. Lett. 95 (2005) 17201; Y. Ohno, et al., Nature 402 (1999) 790.

[14] T. Darnhofer, and U. Rössler, Phys. Rev. B. 47 (1993) 16020.

[15] T. Darnhofer, U. Rössler, and D.A. Broido Phys. Rev. B. 53 (1996) 13631.

[16] F.B. Pedersen, Y.C Chang, Phys. Rev B 55 (1997) 4580.

[17] D. Reuter, P. Kailuweit, A.D. Wieck, U. Zeitler, O. Wibbelholf, C. Meier, A. Lorke, J.C. Maan, Phys. Rev.
Lett. 94 (2005) 026808; D. Reuter, P. Kailuweit, A.D. Wieck, U. Zeitler, J.C. Maan, Physica E 26 (2005) 446.

[18] J.H. Blokland, et al., Phys. Rev. B 75 (2007) 233305.

[19] S. Roddaro, et al., Phys. Rev. Lett. 101 (2008) 186801; F.A. Zwanenburg, et al., Nano Lett. 9 (2009) 1071.

[20] D.M. Gvozdic and U. Ekenberg, Europhys. Lett. 73 (2006) 927.

[21] O. Mauritz and U. Ekenberg, Phys. Rev. B 55 (1997) 10729.

[22] B. Habib, et al., Appl. Phys. Lett. 85 (2004) 3151.

[23] X.W. Zhang, J.B. Xia, J. Phys. D: Appl. Phys. 40 (2007) 541.

[24] J.M. Luttinger, Phys. Rev. 102 (1956) 1030.

[25] M. Fricke, et al., Europhys. Lett. 36 (1996) 197.

[26] R. Winkler, Spin-orbit coupling effects in twodimmensional electron and hole systems (Springer-Verlag, Berlin 2003).

[27] P.C. Sercel, K.J. Vahala. Phys. Rev. B 42 (1990) 3690.

[28] I. Vurgaftman, I.R. Meyer, L.R. Ram-Mohan, J. Appl. Phys. 89 (2001) 5815.

[29] R.J. Warburton, et al., Phys. Rev. B 58 (1998) 16221.

[30] Hong-Yi Chen, V. Apalkov, T. Chakraborty, Phys. Rev. $B 75$ (2007) 193303; Hong-Yi Chen, P. Pietiläinen, T. Chakraborty, Phys. Rev. B 78 (2008) 073407. 